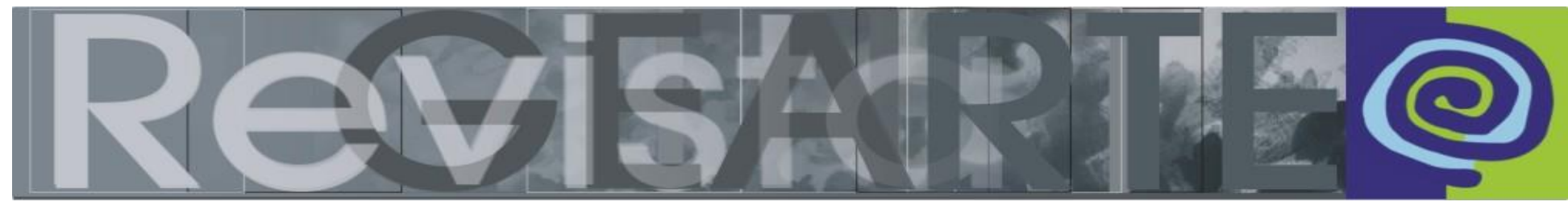

ISSN 2357-9854 | e-ISSN 2596-3198 (online)

\title{
Por una cultura material cíclica desde el arte y la artesanía
}

\author{
Lucía Loren Atienza \\ (Universidad Nebrija y Instituto Nebrija de Competencias Profesionales, Madrid, España)
}

RESUMEN - Por una cultura material cíclica desde el arte y la artesanía - El desarrollo de mi práctica artística, en estos últimos años, ha derivado hacia una búsqueda y comprensión del propio territorio como generador de ecosistemas naturales y culturales que nos vinculan de modo muy estrecho al lugar. Conocer este lugar y partir de él es una premisa fundamental a la hora de realizar una intervención artística en el paisaje. Entiendo este proceso de creación como una colaboración con el ecosistema: el árbol, la tierra, el agua, los animales y, por extensión, el campesino, el artesano o el pastor, son también parte activa y transformadora de la propia obra. El proceso de trabajo en la naturaleza me pone en contacto con la realidad cotidiana, no solo del paisaje, sino de las personas que viven en él, sus privilegios y sus dificultades. Utilizar los propios materiales del entorno se convierte en una herramienta de investigación sobre la que trabajar, para comprender el proceso cíclico de continuas transformaciones sobre el que se dibuja el paisaje. La artesanía tradicional, la agricultura ecológica y el pastoreo extensivo han sido grandes fuentes de inspiración en mi proceso de trabajo, revalorizando una cultura material cíclica, que parte de los propios recursos del territorio sin generar residuos ajenos. La relación con los habitantes de los entornos en los que trabajo suele ser muy estrecha. En ocasiones, los procesos de trabajo son participativos o se generan propuestas de colaboración.

\section{PALABRAS CLAVE}

Arte. Artesanía. Paisaje. Ecología.

RESUMO -- Por uma cultura material cíclica da arte e do artesanato - O desenvolvimento da minha prática artística, nos últimos anos, tem levado à busca e compreensão do próprio território como gerador de ecossistemas naturais e culturais que nos ligam intimamente ao lugar. Conhecer este lugar e a partir dele é uma premissa fundamental na realização de uma intervenção artística na paisagem. Entendo este processo criativo como uma colaboração com o ecossistema: a árvore, a terra, a água, os animais e, por extensão, o camponês, o artesão ou o pastor, são também uma parte ativa e transformadora do 


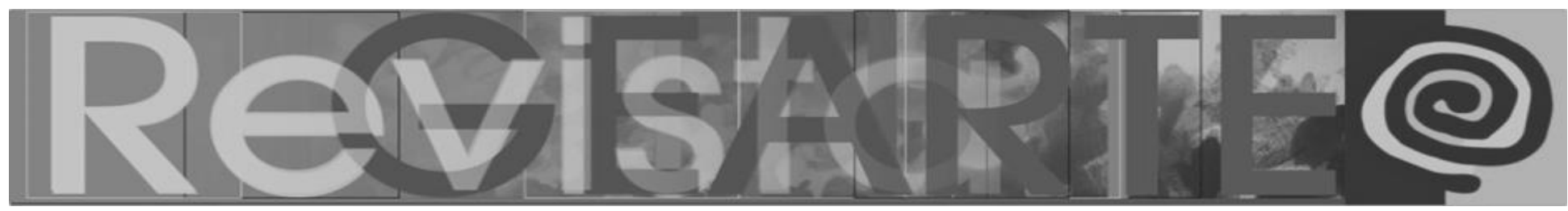

próprio trabalho. O processo de trabalho na natureza me coloca em contato com a realidade cotidiana, não só da paisagem, mas das pessoas que nela vivem, seus privilégios e suas dificuldades. Usar os materiais do próprio ambiente torna-se uma ferramenta de pesquisa para trabalhar, para entender o processo cíclico de transformações contínuas sobre o qual a paisagem é desenhada. O artesanato tradicional, a agricultura orgânica e o pastoreio extensivo têm sido grandes fontes de inspiração no meu processo de trabalho, revalorizando uma cultura material cíclica, que parte dos recursos próprios do território sem gerar resíduos externos. A relação com os moradores dos ambientes em que trabalho costuma ser muito próxima. Às vezes os processos de trabalho são participativos ou propostas colaborativas são geradas.

\section{PALAVRAS-CHAVE}

Arte. Artesania. Paisagem. Ecologia.

ABSTRACT - For a cyclical material culture from art and crafts - The development of my artistic practice, in recent years, has led to a search and understanding of the territory itself as a generator of natural and cultural ecosystems that link us in a very close way to the place. Knowing this place and starting from it is a fundamental premise when carrying out an artistic intervention in the landscape. I understand this creative process as a collaboration with the ecosystem: the tree, the land, the water, the animals and, by extension, the peasant, the artisan or the shepherd, are also an active and transforming part of the work itself. The work process in nature puts me in contact with the daily reality, not only of the landscape, but of the people who live in it, their privileges and their difficulties. Using the materials of the environment itself becomes a research tool to work on, to understand the cyclical process of continuous transformations on which the landscape is drawn. Traditional crafts, organic farming and extensive grazing have been great sources of inspiration in my work process, revaluing a cyclical material culture, which starts from the territory's own resources without generating foreign waste. The relationship with the inhabitants of the environments in which I work is usually very close. Sometimes the work processes are participatory or collaborative proposals are generated.

\section{KEYWORDS}

Art. Crafts. Landscape. Ecology. 


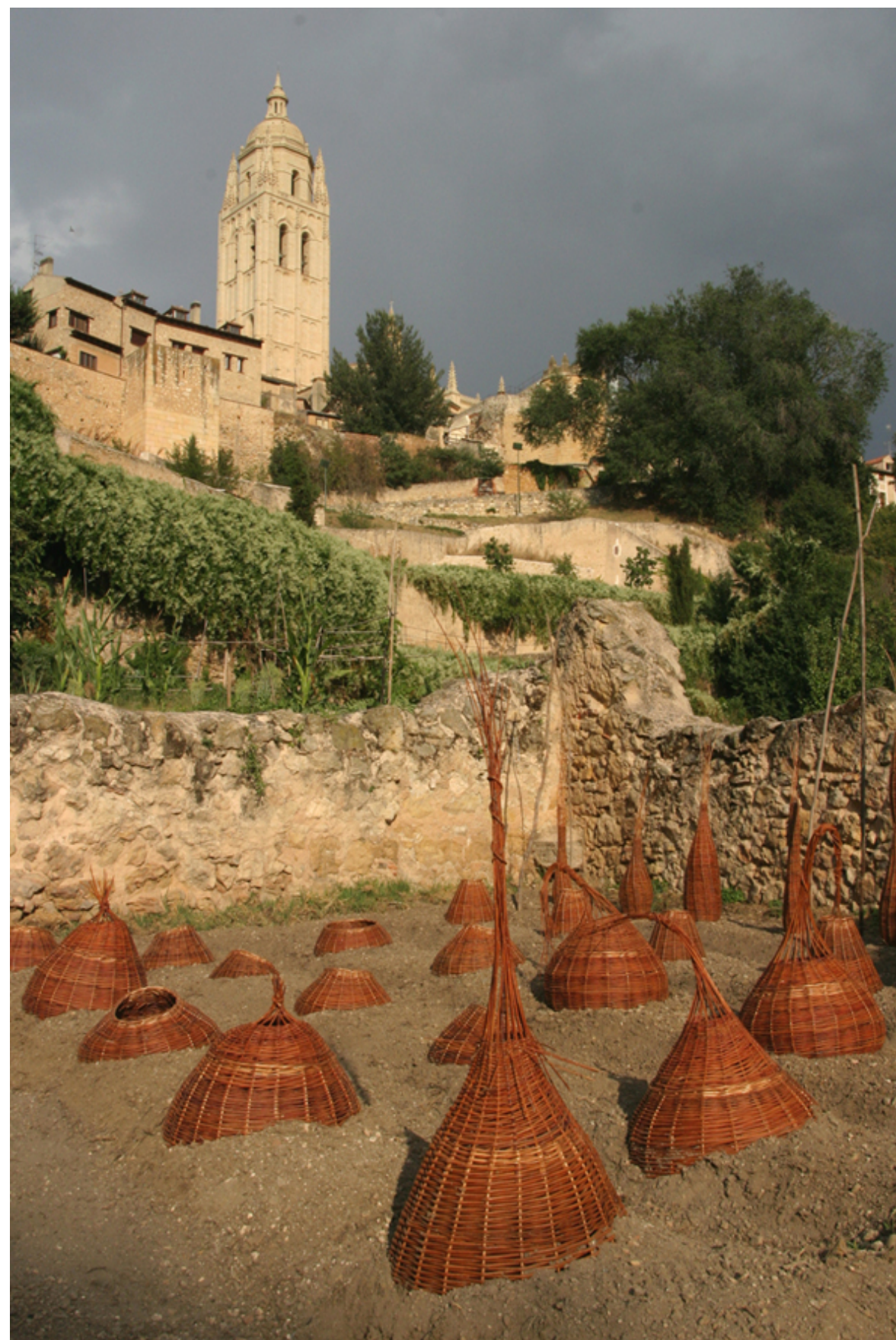

arqueología de una huerta

"Naturalmente Artificial el arte español y la naturaleza, 1968-2006"

museo arte contemporáneo Esteban Vicente, Segovia, 2006

Esta intervención pretende señalar otra forma de relación entre los habitantes de Segovia y su paisaje, concretamente con sus tierras cultivables: "las huertas", indispensables para el desarrollo de la ciudad y la subsistencia de sus pobladores. Un paisaje humano, antropizado (modificado por su acción) y, por tanto, un paisaje cultural. La obra se desarrolla en el interior de una ruina, en una zona de huertas, donde se ha desbrozado el terreno, distribuyendo y preparando los bancales y surcos, reactivando por tanto la relación hombre-tierra. En ese espacio, que ya ha sido absorbido por la ciudad, se han sembrado unos canastos de formas orgánicas que han sido tejidos con mimbre. Se trata de una huerta simbólica, donde los cestos se transforman en una huella fósil de una cosecha imaginaria. Al finalizar el periodo de la intervención un hortelano pasará a cultivar este espacio. 


\section{matriz de agua}

I Festival Internacional Arte en la Viña,

Bodegas Aroa, Navarra, 2017

"El agua es el alma madre de la vida y la matriz, no hay vida sin agua"

\section{ALBERT SZENT GYORGI}

La matriz es el vaso hermético del alquimista, lugar de transformación donde se unen los opuestos: tierra y agua, muerte y renacimiento. Símbolo del órgano sexual femenino, espacio tejido para acoger los frutos, vientre contenedor de las cosechas recién recogidas.

más info: http://www.aroawines.com/arteenlavina/

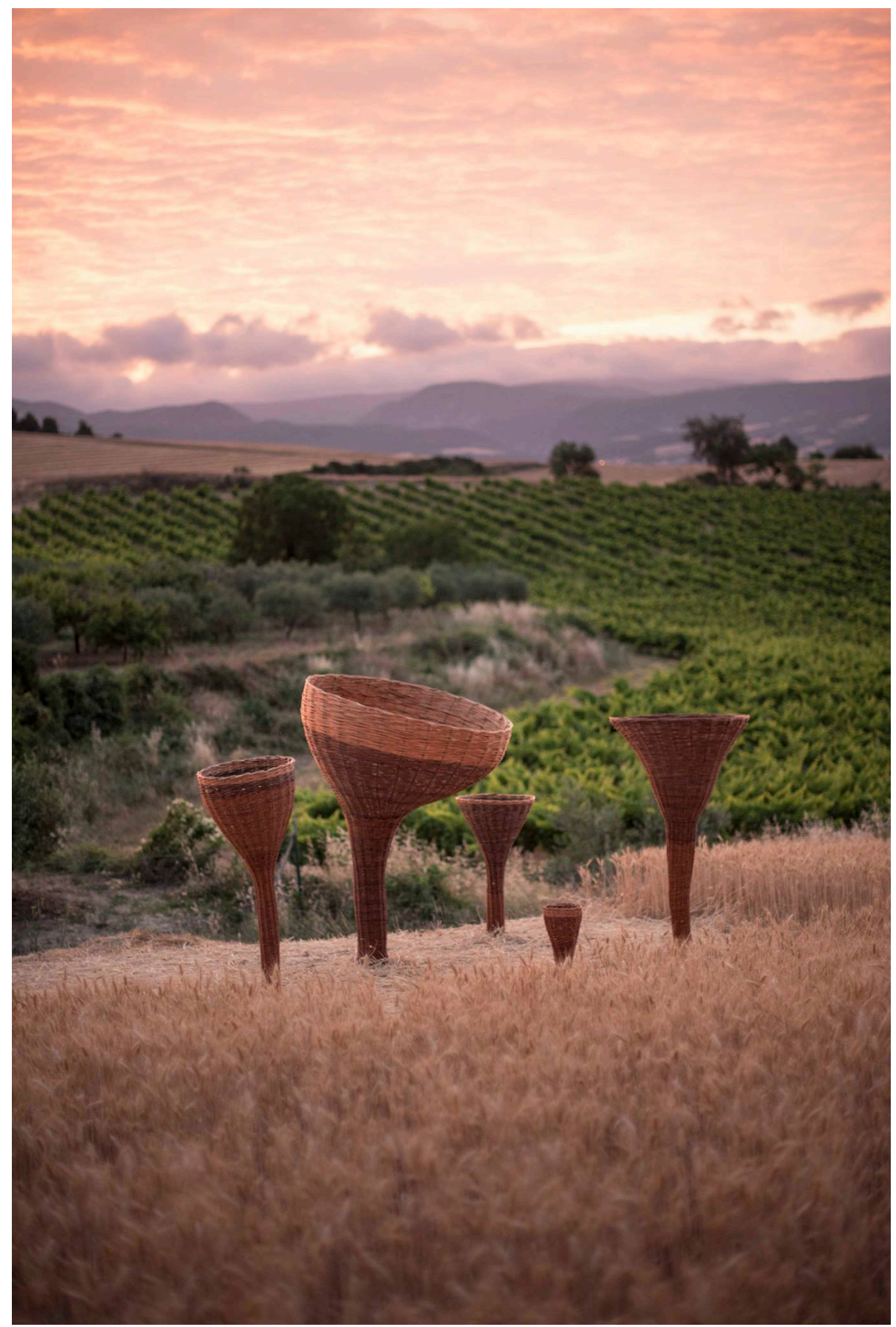




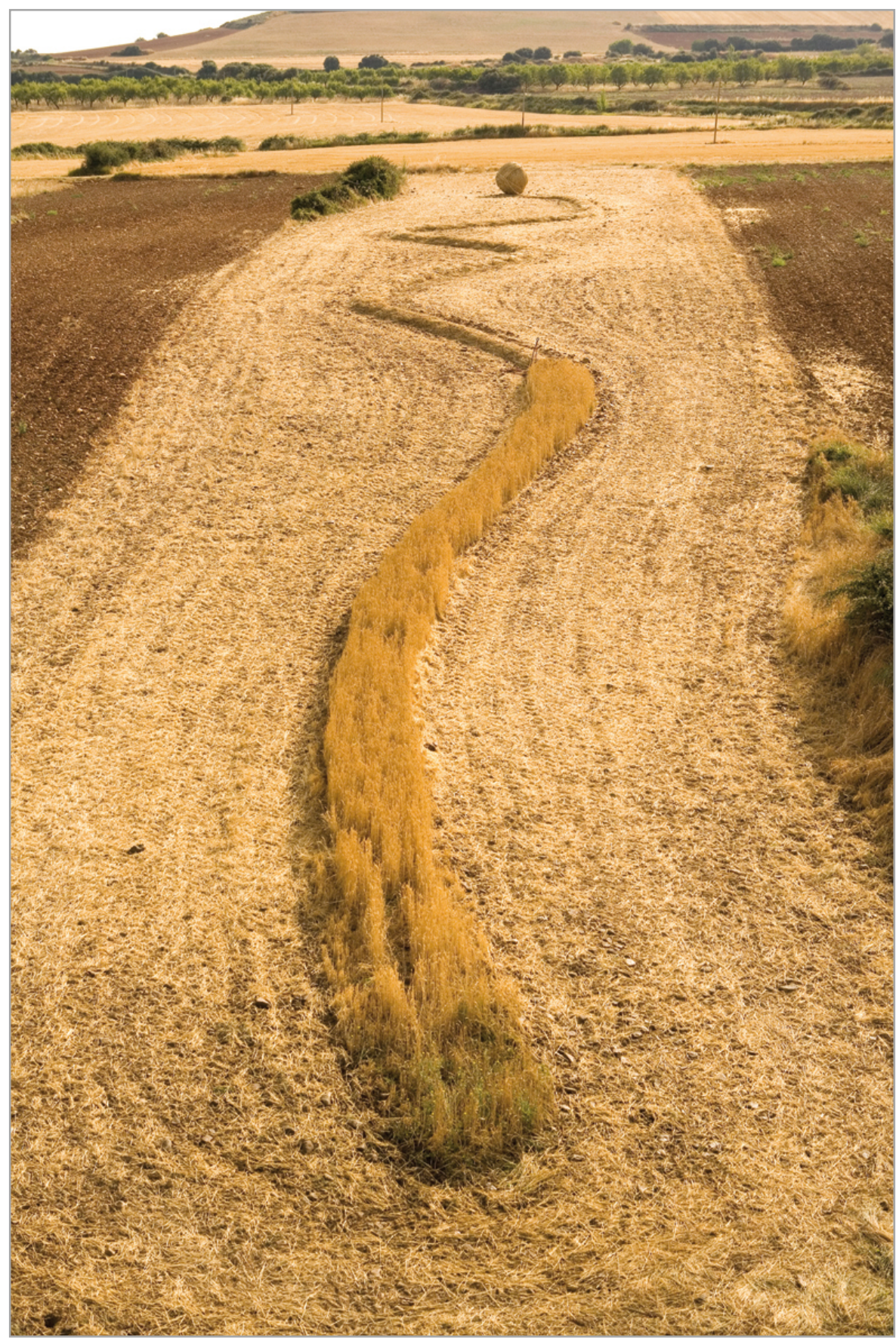

\section{al hilo del paisaje}

"arte en la tierra"

Santa Lucía de Ocón

La Rioja, 2007

Un gran ovillo de trigo se une al campo de cultivo a partir de una hebra. En esta acción, el cosido se presenta como un proceso de reunificación simbólica con la tierra. Una reflexión sobre la relación entre el espacio cultivado y el espacio tejido. Una metáfora sobre el paisaje convertido en un gran manto parcheado de cultivos. Paisajes de diversidad y equilibrio, paisajes del cuidado por la vida.

Esta pieza se realizó con la generosa colaboración de los habitantes de Santa Lucía de Ocón, que desde le inicio de la convocatoria se han implicado activamente hasta consolidar un proyecto de arte completamente integrado en su comunidad y que es toda una referencia a nivel nacional. 


\section{Ombra}

Art in Bosco, 2019

"Ombra", una esfera realizada en mimbre alrededor del tronco de un joven haya, se inspira en la trama natural que genera el hayedo para filtrar e impedir el paso directo de la luz a la corteza, ya que los rayos directos del sol le ocasionarían grietas y quemaduras que pueden promover su deterioro. "Ombra" acompaña, con su estructura esférica alrededor al tronco del árbol, este abrazo sombrío y cuidador.

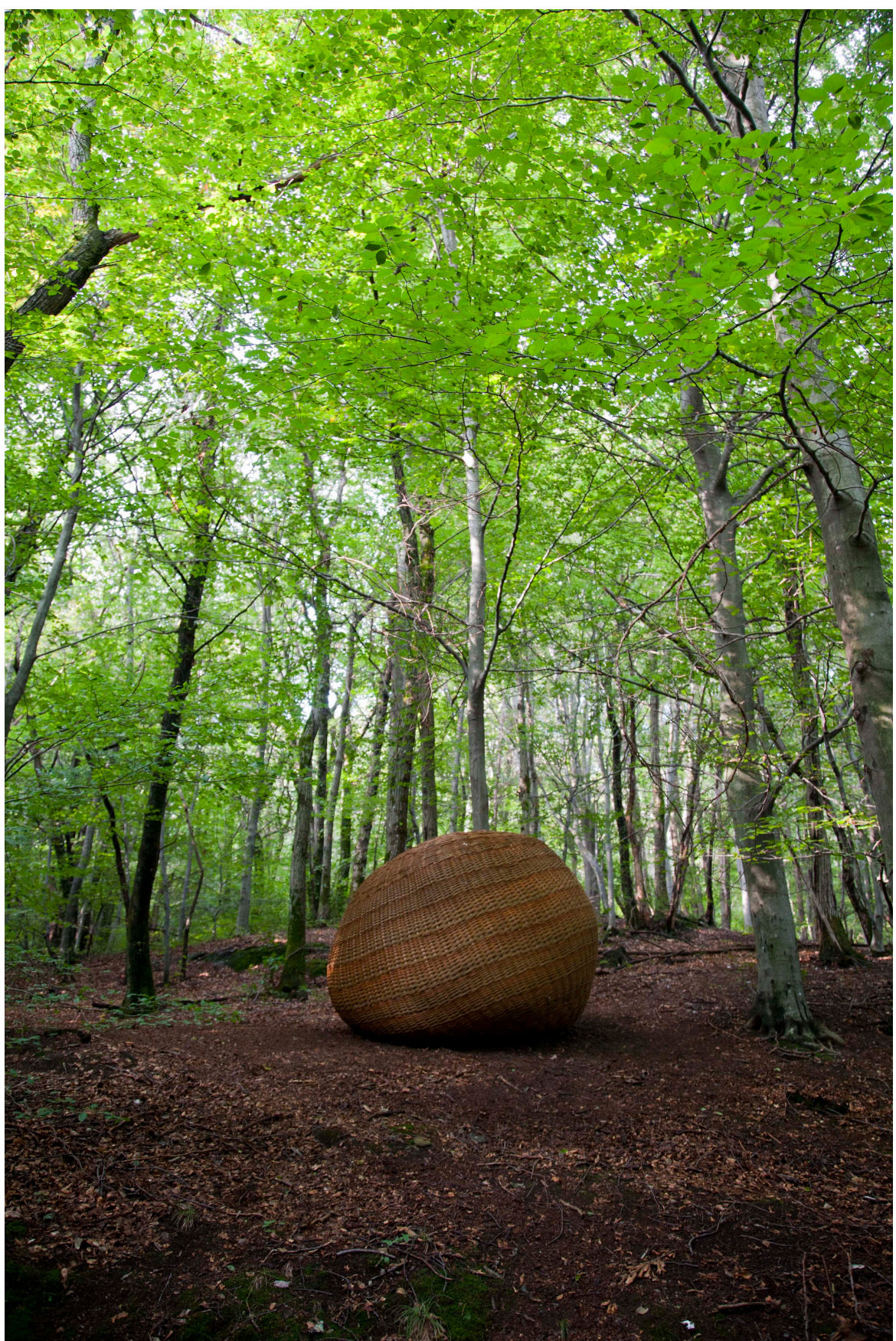




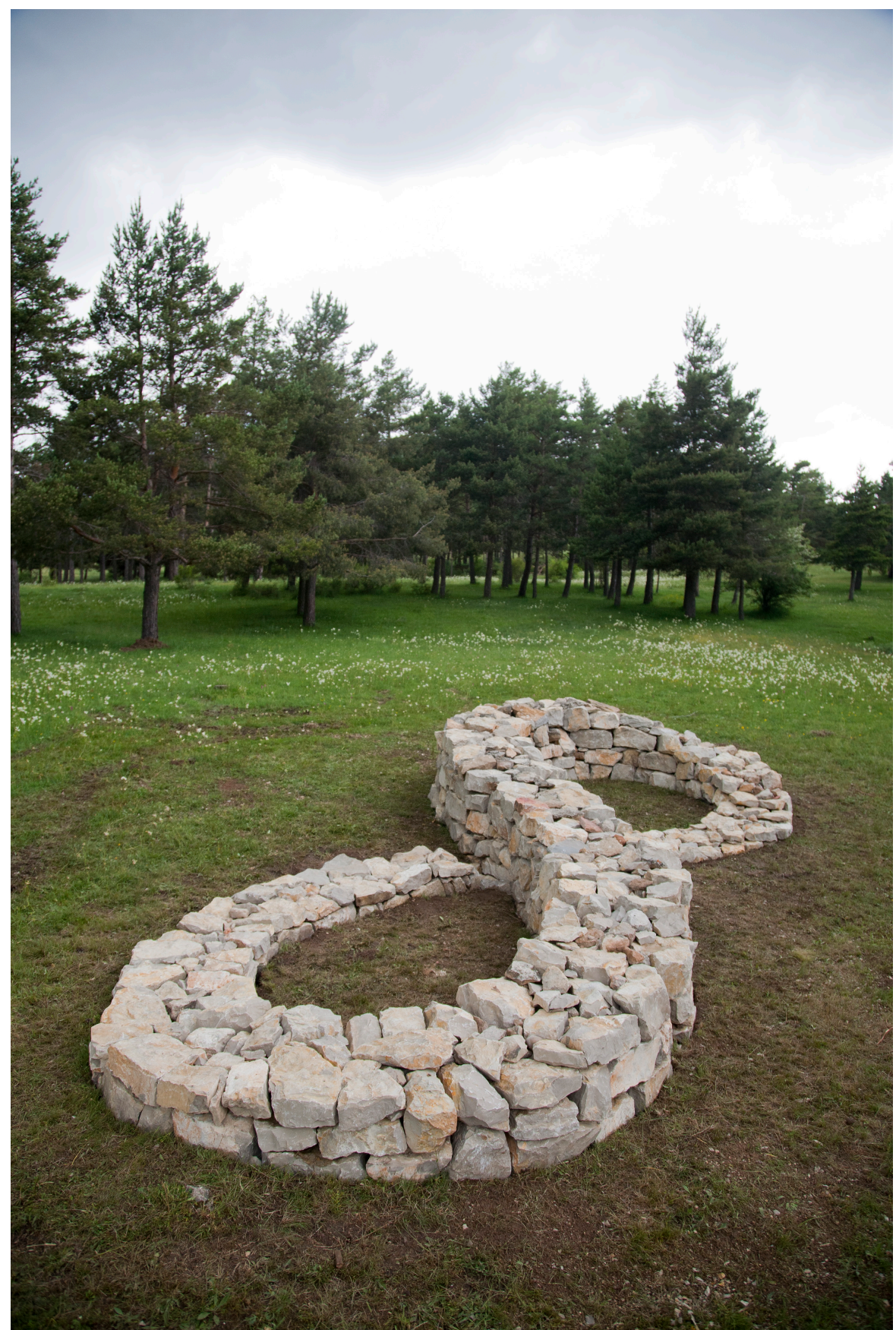

El concepto de deslinde, hace referencia a los límites de un terreno, acotando el lugar físico de la propiedad privada. Los muros de piedra seca, mojones y otros elementos similares, han formado parte de una arquitectura vernácula vinculada con estas demarcaciones territoriales. Desde la Edad Media, el entorno de Guadalaviar ha sido un territorio muy complejo, inmerso en continuas hostilidades a raíz de los conflictos territoriales derivados de los aprovechamientos comunales y los procesos de deslindes. Esta intervención dibuja un infinito de piedra, en un intento imposible de contener lo intangible, de atrapar lo que no tiene límites.

Esta intervención pretende activar el interés por una forma de construcción tan importante como la piedra seca, muy significativa en esta zona de fuerte cultura pastoril y actividad transhumante.

Es destacable señalar el valor ambiental de esta técnica de construcción artesana, que procura corredores ecológicos fundamentales para diversos hábitats, así como refugio de flora y fauna primordial utilizando los propios recursos del lugar.

En el proyecto de creación de la pieza, han colaborado habitantes del pueblo y de fuera, dando forma a la intervención en un taller asesorado por el equipo técnico de XEROpiedra 100\%. Artesanía. 


\section{Lucía Loren Atienza}

Licenciada Bellas Artes, Universidad Complutense de Madrid. Docente del Grado de Bellas Artes de la Universidad Nebrija y en el Instituto Nebrija de Competencias Profesionales. Pertenece al Grupo Nebrija de Estudios Transversales en Creación Contemporánea ETCC. Docente MIAM, Máster Oficial Investigaciones Artes, Música y Educación Estética, UJA.

Mi línea de investigación gira en torno a las prácticas de arte contemporáneo que integran una reflexión socioambiental de nuestra relación con el territorio, articulando praxis colaborativas, procesos de intervención social e integrando estas experiencias en contextos educativos inclusivos.

www.lucialoren.com
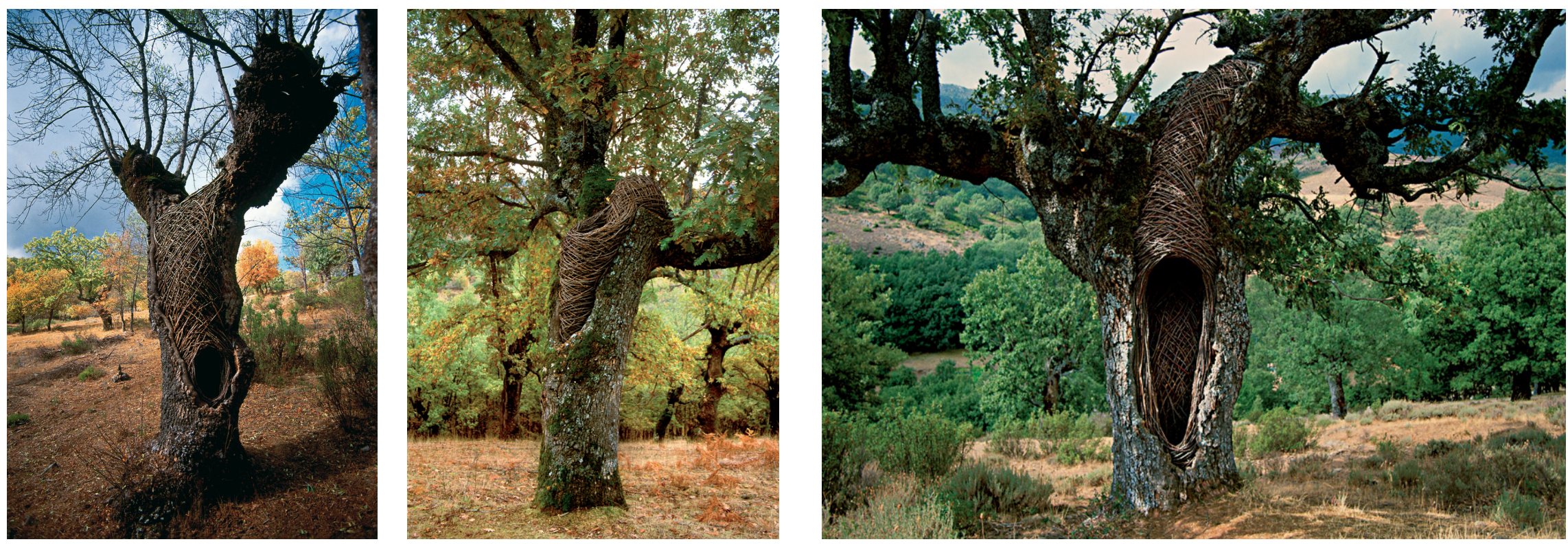
Licenciada Bellas Artes, Universidad Complutense de Madrid. Docente del Grado de Bellas Artes de la Universidad Nebrija y en el Instituto Nebrija de Competencias Profesionales. Pertenece al Grupo Nebrija de Estudios Transversales en Creación Contemporánea ETCC. Docente MIAM, Máster Oficial Investigaciones Artes, Música y Educación Estética, UJA.

Mi línea de investigación gira en torno a las prácticas de arte contemporáneo que integran una reflexión socioambiental de nuestra relación con el territorio, articulando praxis colaborativas, procesos de intervención social e integrando estas experiencias en contextos educativos inclusivos.
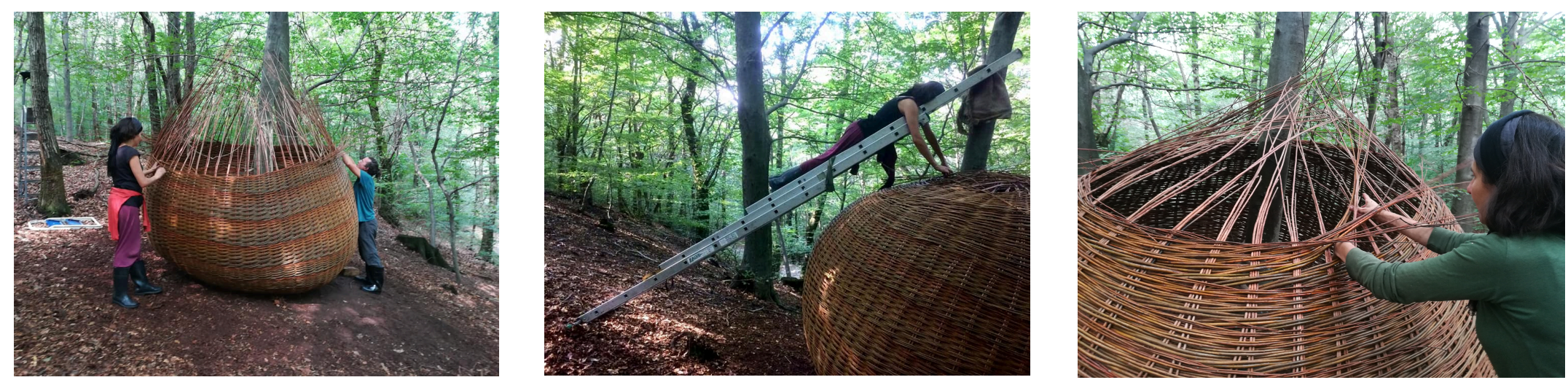

Fotografías proceso intervención "ombra" Mimbre 


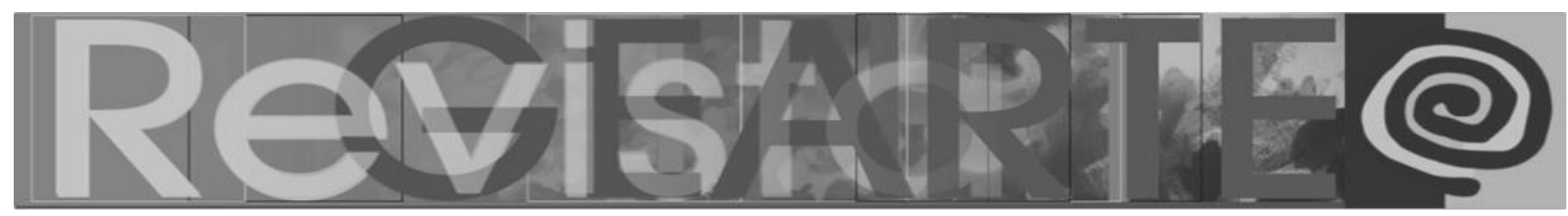

\section{Lucía Loren Atienza}

Licenciada Bellas Artes, Universidad Complutense de Madrid. Docente del Grado de Bellas Artes de la Universidad Nebrija y en el Instituto Nebrija de Competencias Profesionales. Pertenece al Grupo Nebrija de Estudios Transversales en Creación Contemporánea ETCC. Docente MIAM, Máster Oficial Investigaciones Artes, Música y Educación Estética, UJA. Mi línea de investigación gira en torno a las prácticas de arte contemporáneo que integran una reflexión socio- ambiental de nuestra relación con el territorio, articulando praxis colaborativas, procesos de intervención social e integrando estas experiencias en contextos educativos inclusivos.

ORCID: https://orcid.org/0000-0002-0456-3113

E-mail: lucialoren@yahoo.com

Currículo: http://www.lucialoren.com

Recebido em 29 de maio de 2020 Aceito em 18 de agosto de 2020 